\title{
A 6-year open-label study of the efficacy
} and safety of olanzapine long-acting injection in patients with schizophrenia: a post hoc analysis
based on the European label recommendation

This article was published in the following Dove Press journal:

Neuropsychiatric Disease and Treatment

27 May 2015

Number of times this article has been viewed

\section{Ernie Anand' \\ Lovisa Berggren² \\ Claudia Deix ${ }^{3}$ \\ Ágoston Tóth ${ }^{4}$ \\ David P McDonnell ${ }^{5}$}

'Neuroscience Medical Affairs - EU, Eli Lilly, Windlesham, United Kingdom; ${ }^{2}$ Global Statistical Sciences, Lilly Deutschland GmbH, Bad Homburg, Germany; ${ }^{3}$ Neuroscience, Eli Lilly Regional Operations GmbH,Vienna, Austria; ${ }^{4}$ Lilly Hungary, Budapest, Hungary; ${ }^{5}$ Lilly Research Laboratories, Eli Lilly and Company, Indianapolis, IN, USA
Correspondence: Ernie Anand

Neuroscience Medical Affairs - EU, Lilly

Research Centre, Erl Wood Manor,

Windlesham, Surrey, GU20 6PH,

United Kingdom

Tel +44 I2 76483063

Fax +44 I2 7648 37II

Email anand_ernie@lilly.com
Purpose: To assess the long-term efficacy and safety of olanzapine long-acting injection (LAI) in the treatment of schizophrenia, focusing on clinical trial data consistent with the approved indication and dosing recommendations in the European label and which forms the basis for treatment decisions made by clinicians in daily clinical practice.

Patients and methods: This was a post hoc analysis of a 6-year open-label study of olanzapine LAI in patients (male or female, 18-75 years old) with schizophrenia entering this study following feeder studies of olanzapine LAI. Patients were flexibly dosed (45-405 mg, 2- to 4-week intervals), but those receiving oral olanzapine supplementation whose total olanzapine dose was $>20 \mathrm{mg}$ /day equivalent were excluded from this post hoc analysis.

Results: Data from 669 patients were analyzed (44.5\% completed). Positive and Negative Syndrome Scale total scores did not change significantly from baseline to endpoint; Clinical Global Impression-Severity scores improved significantly. Mean weight change was $+2.19 \mathrm{~kg}$ ( $P<0.001)$, with $40.8 \%$ of patients experiencing $\geq 7 \%$ weight gain. There were 24 occurrences of post-injection delirium/sedation syndrome (PDSS).

Conclusion: Olanzapine LAI appeared to be effective in the long-term maintenance of schizophrenia, and the safety profile was consistent with that of oral olanzapine, except for injection-related events and PDSS events.

Keywords: antipsychotic, depot, long-acting injection, olanzapine, pamoate, schizophrenia

\section{Introduction}

In an attempt to improve the long-term treatment of schizophrenia, depot antipsychotic medications were developed beginning in the 1960s. ${ }^{1}$ However, challenges of adherence and concomitant positive long-term outcomes still remain. The recent introduction of depot formulations of the second-generation antipsychotics, or atypicals, into clinical practice may be helping to address some of these clinical challenges, and, correspondingly, this is reflected in recent treatment guidelines. ${ }^{2}$ One such secondgeneration antipsychotic depot formulation is that of olanzapine (the pamoate salt of olanzapine; olanzapine long-acting injection [LAI]), whose efficacy and safety in the treatment of schizophrenia has been previously documented. ${ }^{3-8}$ Those studies, however, were of relatively short duration. Studies that evaluate the long-term profile of depot antipsychotics in the treatment of schizophrenia are clearly going to be more reflective of clinical reality, ${ }^{9}$ and recently a 6-year, single-arm, open-label extension study of olanzapine LAI assessing its safety and efficacy was published. ${ }^{10}$ That study, however, was designed and implemented before olanzapine LAI was approved for clinical use, 
and allowed patients with schizoaffective disorder and total olanzapine doses above an equivalent of $20 \mathrm{mg} /$ day, both of which are not consistent with the current recommendations of the European label for olanzapine LAI. ${ }^{11}$

The present report is of a post hoc analysis of the McDonnell et $\mathrm{al}^{10}$ study, the rationale for which is to ascertain if there would be any clinical or safety differences in the use of olanzapine LAI in a patient population defined in the recommendations of the European label. All other analyses presented were prespecified and are consistent with the original study protocol.

\section{Materials and methods}

\section{Patients and design}

The original study, ${ }^{10}$ from which the data for this post hoc analysis were derived, was a multinational, multicenter, single-arm, open-label phase 3 study of olanzapine LAI in patients with schizophrenia and schizoaffective disorder conducted at 127 sites in 25 countries. Patients were enrolled starting in August 2004, and although the total duration was 6 years and 4 months, patients were permitted to continue treatment in the study only until such time as the product was commercially available in their country (as early as 2009) or until December 31, 2010, whichever came first. Patients could enroll immediately following their completion in participating in any of three feeder studies: F1D-MC-HGJZ, ${ }^{6}$ F1D-MCHGKA, ${ }^{5}$ or F1D-EW-LOBS (Eli Lilly and Company, Ltd, data accessed April 21st, 2015). Study F1D-EW-LOBS was a 4-week pharmacokinetic study of olanzapine LAI $(\mathrm{N}=134)$ in which patients with schizophrenia or schizoaffective disorder were first stabilized on oral olanzapine (5-20 mg/day) and then given a single injection of $405 \mathrm{mg}$ olanzapine LAI. A single $5 \mathrm{mg}$ dose of olanzapine rapid-acting intramuscular injection was then given 26 days later.

For this post hoc analysis, patients were male or female, between 18 years and 75 years of age who met diagnostic criteria for schizophrenia according to the Diagnostic and Statistical Manual of Mental Disorders, 4th edition, text revision (DSM-IV-TR). ${ }^{12}$ (Because of the nature of the feeder studies, the majority of patients were not acutely psychotic at study entry). For the purposes of this post hoc analysis, to be consistent and in accordance with the current recommendations of the European label, patients with schizoaffective disorder were excluded. All patients exhibited acceptable results on clinical laboratory tests, vital signs, and electrocardiograms at screening.

\section{Dosing}

In the original McDonnell et al ${ }^{10}$ study, patients were permitted to receive supplemental oral olanzapine up to
$20 \mathrm{mg} /$ day and the maximum total dose of olanzapine LAI permitted was $600 \mathrm{mg}$ over 4 weeks. In this post hoc analysis, we excluded those patients who received oral olanzapine supplementation who had a total daily dose of more than $20 \mathrm{mg} /$ day oral olanzapine equivalent. Thus, the doses and intervals allowed in this post hoc analysis are consistent and in accordance with the current recommendations of the European label. ${ }^{11}$

\section{Efficacy}

Efficacy was assessed using mean change on the Positive and Negative Syndrome Scale (PANSS), ${ }^{13}$ every 6 months and at discontinuation. The Clinical Global Impression-Severity (CGI-S) of illness was administered at each visit to assess mean change from baseline over time. ${ }^{14}$

\section{Health outcomes/quality of life}

The Patient Satisfaction with Medication Questionnaire (PSMQ) uses a Likert-type scale (scored 1-5) to assess patient satisfaction with antipsychotics. ${ }^{15}$ The PSMQ-Modified (PSMQ-Mod) includes three additional items: satisfaction with current depot medication, preference between current depot medication and previous oral medications, and the comparison of side effects between current depot medication and previous oral medications. The PSMQ-Mod was performed every 6 months and at endpoint and is summarized by item.

To provide information about hospitalizations, the total number of days spent in the hospital per patient-year of exposure to treatment was computed. The percentage of patients with hospitalizations was also computed.

\section{Safety}

Treatment-emergent adverse events (TEAEs), serious adverse events (SAEs), and adverse events (AEs) leading to study discontinuation; mean changes in vital signs and weight; mean changes and treatment-emergent categorical changes in fasting glucose, fasting lipids, and other laboratory measures; and categorical changes in weight and electrocardiogram measurements are all summarized.

Extrapyramidal symptoms (EPS) were assessed using the Simpson-Angus Scale (SAS), ${ }^{16}$ the Barnes Akathisia Scale (BAS), ${ }^{17}$ and the Abnormal Involuntary Movement Scale (AIMS) ${ }^{14}$ at the first open-label visit, at each 6-month visit, and at the discontinuation visit. Analyses were conducted to evaluate the proportion of patients with treatment-emergent Parkinsonism (SAS total score $>3$ at any visit), akathisia (BAS global score $\geq 2$ at any visit), and abnormal dyskinetic movements (score $\geq 3$ for any of AIMS items $1-7$ or a score $\geq 2$ for any two of these items). 
As a result of the possibility of the occurrence of postinjection delirium/sedation syndrome (PDSS) events after an injection of olanzapine LAI, the protocol was amended approximately 2 years after the start of the study to require patients to be observed at the health care facility for 3 hours after receiving an injection of olanzapine LAI. ${ }^{3,10}$

\section{Statistics}

As stated previously, all patients who received supplemental doses of oral olanzapine whose total daily dose of olanzapine was $>20 \mathrm{mg} /$ day equivalent were excluded from all analyses. Also, all patients with schizoaffective disorder were excluded from this post hoc analysis. Therefore, of the 931 patients included in the original McDonnell et a ${ }^{10}$ study, 262 were excluded. The remaining 669 patients all received at least one dose of olanzapine LAI and were included in the primary safety analyses for this post hoc analysis. For the analysis of efficacy, patients were included only if they had a baseline and postbaseline measure. Baseline was defined as the last observation prior to receiving the injection either at the first open-label visit or the last observation from the feeder study; and endpoint measure was defined as last measure in the study. All hypotheses were tested using a two-sided significance level of 0.05 . Total scores were considered as missing if any of the individual items were missing. For the analysis of continuous measures, missing data were handled using last-observation-carried-forward (LOCF) change from baseline-to-endpoint analyses. Within-group changes from baseline were tested using paired $t$-tests. A mixed-model repeated measures (MMRM) analysis was used for the graphical presentation of PANSS scores. Analyses with categorical factors (eg, sex, race) employed between-group $P$-values from analysis of variance (ANOVA) models. Time to discontinuation was evaluated by Kaplan-Meier survival analysis. No adjustment for multiplicity was done. All analyses were conducted using SAS software (version 9.1.3; SAS Institute, Cary, NC, USA).

\section{Results}

\section{Patients}

Of the 931 patients included in the original McDonnell et a ${ }^{10}$ study, 669 were treated with olanzapine LAI in a manner that is consistent with the current recommendations of the European label, and were therefore included in this post hoc analysis. Patient demographics and baseline characteristics are presented in Table 1. Of these 669 patients, $66.1 \%$ were male and $67.7 \%$ were Caucasian; mean age was 39.4 years. During the observed period of this post hoc analysis, $47(7.0 \%)$ patients received supplemental oral olanzapine
Table I Patient demographics and baseline characteristics

\begin{tabular}{|c|c|}
\hline Characteristic & $\begin{array}{l}\text { Olanzapine LAl } \\
\text { patients }(\mathrm{N}=669)\end{array}$ \\
\hline Age, years, mean (SD) & $39.4(11.8)$ \\
\hline Sex (male), n (\%) & $442(66.1)$ \\
\hline \multicolumn{2}{|l|}{ Race, n (\%) } \\
\hline Caucasian & $453(67.7)$ \\
\hline Hispanic & $108(16.1)$ \\
\hline African & $60(9.0)$ \\
\hline East Asian & $29(4.3)$ \\
\hline West Asian & $17(2.5)$ \\
\hline Native American & $2(0.3)$ \\
\hline \multicolumn{2}{|l|}{ Number of previous episodes } \\
\hline \multicolumn{2}{|l|}{ or exacerbations of schizophrenia } \\
\hline \multicolumn{2}{|l|}{ in the last 24 months, $\mathrm{n}(\%)$} \\
\hline 0 & $179(26.8)$ \\
\hline I & $209(31.2)$ \\
\hline 2 & $167(25.0)$ \\
\hline 3 & $47(7.0)$ \\
\hline 4 & $27(4.0)$ \\
\hline$\geq 5$ & $40(6.0)$ \\
\hline $\begin{array}{l}\text { Age of onset of first episode of schizophrenia } \\
\text { (years), mean (SD) }\end{array}$ & $25.63(8.8 I)$ \\
\hline $\begin{array}{l}\text { Length of most recent episode of schizophrenia } \\
\text { (days), mean (SD) }\end{array}$ & $784.59(1,644.17)$ \\
\hline PANSS total score, mean (SD) & $52.87(16.43)$ \\
\hline CGI-S, mean (SD) & $2.84(1.01)$ \\
\hline
\end{tabular}

Abbreviations: CGI-S, Clinical Global Impression-Severity; LAI, long-acting injection; $\mathrm{N}$, total number of patients; $\mathrm{n}$, number of affected patients; PANSS, Positive and Negative Syndrome Scale; SD, standard deviation.

(but their total olanzapine dose was not $>20 \mathrm{mg} /$ day equivalent), 211 (31.5\%) used benzodiazepines, and 52 (7.8\%) used anticholinergic agents.

\section{Patient disposition}

The mean duration of patient exposure was 1,110.10 days (approximately 3 years); the longest duration was 2,204 days (approximately 6 years). Overall, olanzapine LAI exposure was 2,033.2 patient years. Patient disposition is presented in Table 2. The most frequently reported AEs leading to discontinuation were schizophrenia (nine patients [1.3\%]), weight increased (seven patients [1.0\%]), and psychotic disorder (four patients [0.6\%]).

\section{Efficacy}

Time to discontinuation is shown in Figure 1;298 patients (44.5\%) remained on olanzapine LAI at the completion of the observed period of this post hoc analysis. Patients on average were "mildly ill"18 at study entry with a mean PANSS total score of 52.87 (SD =16.43) and CGI-S total score of 2.84 ( $\mathrm{SD}=1.01$ ). The baseline-to-endpoint mean change (LOCF) on PANSS total score was -0.26 $(\mathrm{SD}=14.0, P=0.641)$; on PANSS negative score was -0.26 
Table 2 Patient disposition

\begin{tabular}{ll}
\hline $\begin{array}{l}\text { Reason for } \\
\text { discontinuation }\end{array}$ & $\begin{array}{l}\text { Olanzapine LAl patients } \\
(\mathbf{N}=669), \mathbf{n}(\%)\end{array}$ \\
\hline Completed & $298(44.5)$ \\
Patient decision & $194(29.0)$ \\
Adverse event & $47(7.0)$ \\
Lost to follow-up & $45(6.7)$ \\
Physician decision & $25(3.7)$ \\
Sponsor decision & $25(3.7)$ \\
Protocol violation & $14(2.1)$ \\
Lack of efficacy & $13(1.9)$ \\
Death & $8(1.2)$
\end{tabular}

Abbreviations: LAl, long-acting injection; $N$, total number of patients; $n$, number of affected patients.

$(\mathrm{SD}=4.53, P=0.153)$; PANSS positive score was 0.15 ( $\mathrm{SD}=3.92, P=0.350)$; and PANSS general psychopathology total score was -0.15 ( $\mathrm{SD}=7.43, P=0.618)$. A review of individual items of the PANSS score demonstrated small but statistically significant baseline-to-endpoint decreases for active social avoidance $(-0.17, \mathrm{SD}=0.99, P<0.001)$, lack of spontaneity and flow of conversation $(-0.09, \mathrm{SD}$ $=0.83, P=0.009)$, and mannerisms and posturing $(-0.13$, $\mathrm{SD}=0.72, P<0.001)$, and a baseline-to-endpoint increase for poor impulse control $(0.09, \mathrm{SD}=0.81, P=0.007)$. PANSS total scores and subscores over time are presented using an MMRM analysis in Figure 2. Mean improvements from baseline to LOCF endpoint for CGI-S scores were statistically significant $(P \leq 0.02)$ for all measured time points starting at visit 4 (Figure 3 ).

\section{Health outcomes/quality of life}

The PSMQ-Mod was analyzed at endpoint for all patients. The majority of patients responding to this medication satisfaction questionnaire $(\mathrm{N}=620)$ indicated favorable responses for olanzapine LAI compared to previous oral therapy. With the "current depot medication" (olanzapine LAI), $73.7 \%$ of patients ( $\mathrm{n}=457$ ) were "somewhat satisfied" or "very satisfied." Regarding preference of olanzapine LAI versus previous oral medications, $69.5 \%$ of patients $(n=431)$ preferred or much preferred olanzapine LAI. Regarding side effects with olanzapine LAI versus their previous oral therapy, $74.2 \%$ of patients $(n=460)$ thought olanzapine LAI use resulted in "less side effects" or "much less side effects" (Figure 4).

During the observed period of this post hoc analysis, 145 patients $(21.7 \%)$ were hospitalized a total of 153 times. Most $(67 \%)$ of the hospitalizations were for psychiatric reasons.

\section{Safety}

TEAEs reported by $\geq 5 \%$ of the patients are presented in Table 3. During the observed period of this post hoc analysis, $445(66.5 \%)$ patients reported at least one TEAE, of which the five most commonly reported ones were increased weight, somnolence, anxiety, insomnia, and nasopharyngitis. A total of $95(14.2 \%)$ patients reported at least one SAE. The most frequently reported SAEs were schizophrenia $(n=17)$, psychotic disorder $(n=9)$, sedation $(n=7)$, anxiety $(n=4)$, delirium $(n=4)$, agitation $(n=3)$, confusional state $(n=3)$, hypertension

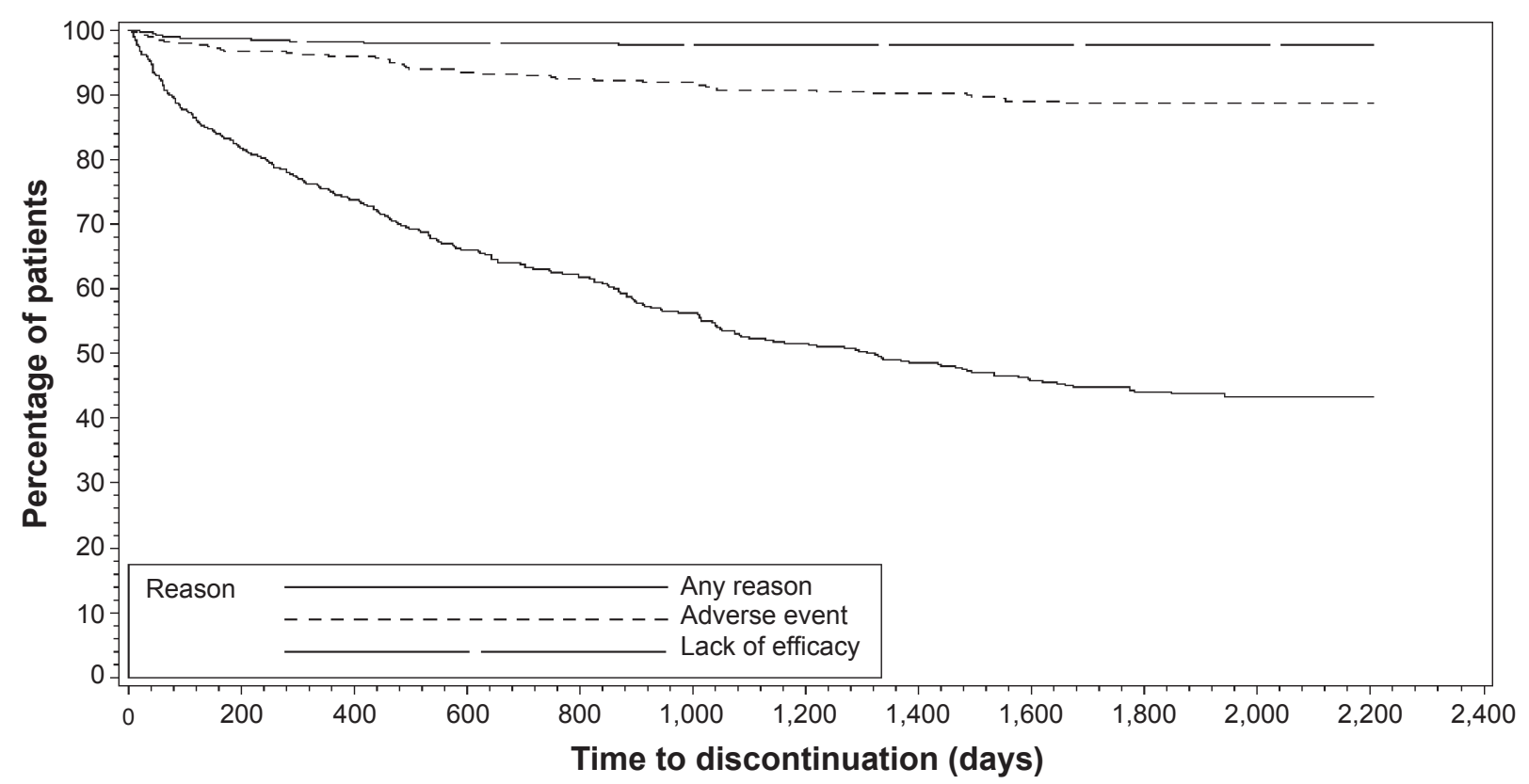

Figure I Kaplan-Meier time to discontinuation ( $\mathrm{N}=669)$.

Abbreviation: $\mathrm{N}$, total number of patients. 

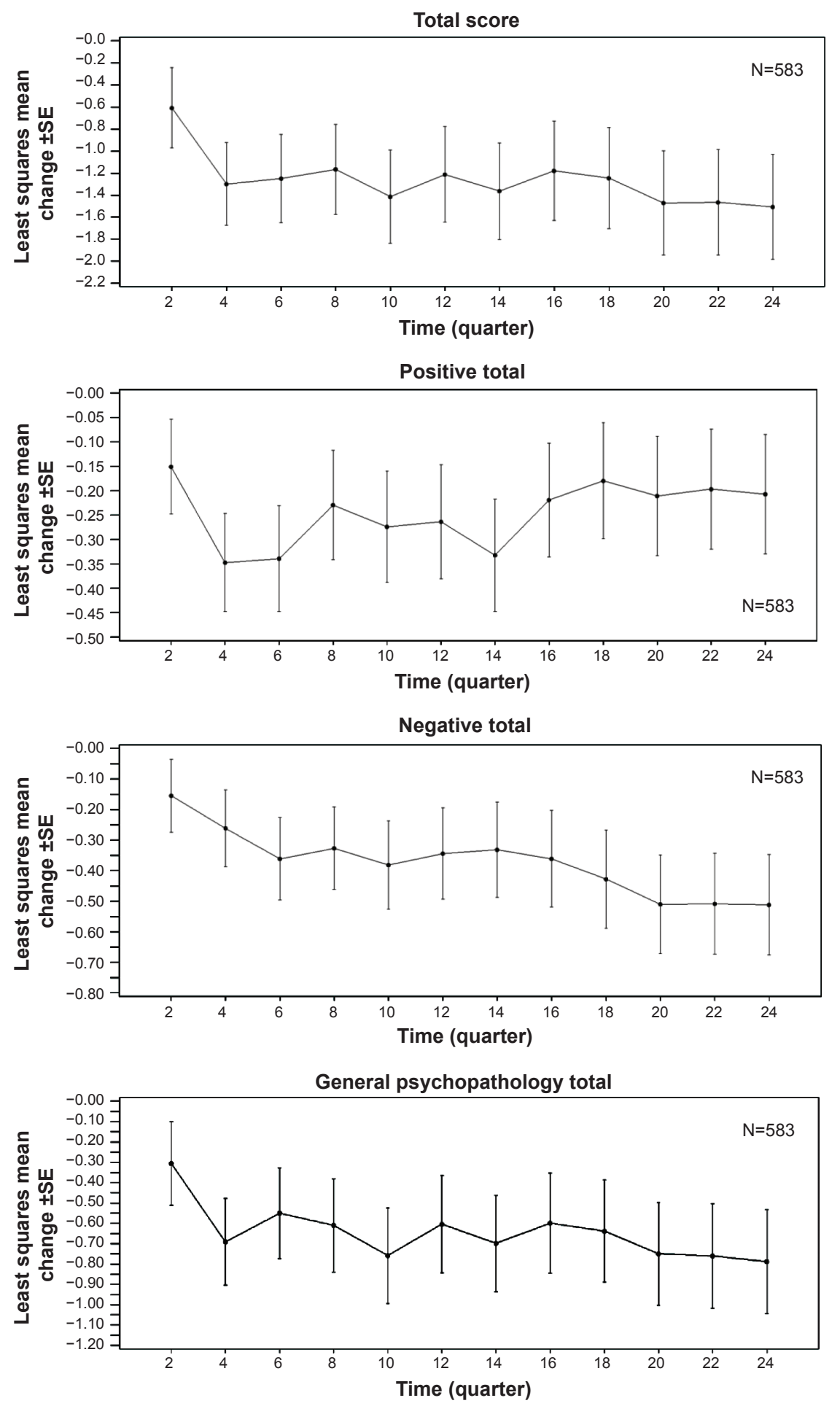

Figure 2 Positive and Negative Syndrome Scale total and subscale scores, change from baseline to endpoint (mixed model repeated measures analysis). Note: A quarter equals 3 months.

Abbreviations: $\mathrm{N}$, number of patients; SE, standard error.

$(n=3)$, myocardial infarction $(n=3)$, paranoia $(n=3)$, and suicidal ideation $(n=3)$.

There were eight deaths, including one suicide, reported during the observed period of this post hoc analysis. Of the eight deaths, seven were not considered by the investigators to be related to treatment with the study drug or to protocol procedures, and one patient's death due to myocardial infarction was considered possibly related to the study drug but not related to protocol procedures. The other causes of death were alcoholic cardiomyopathy, pneumonia, leptospirosis, hypertrophic cardiomyopathy, renal cell carcinoma, essential hypertension, and suicide. 


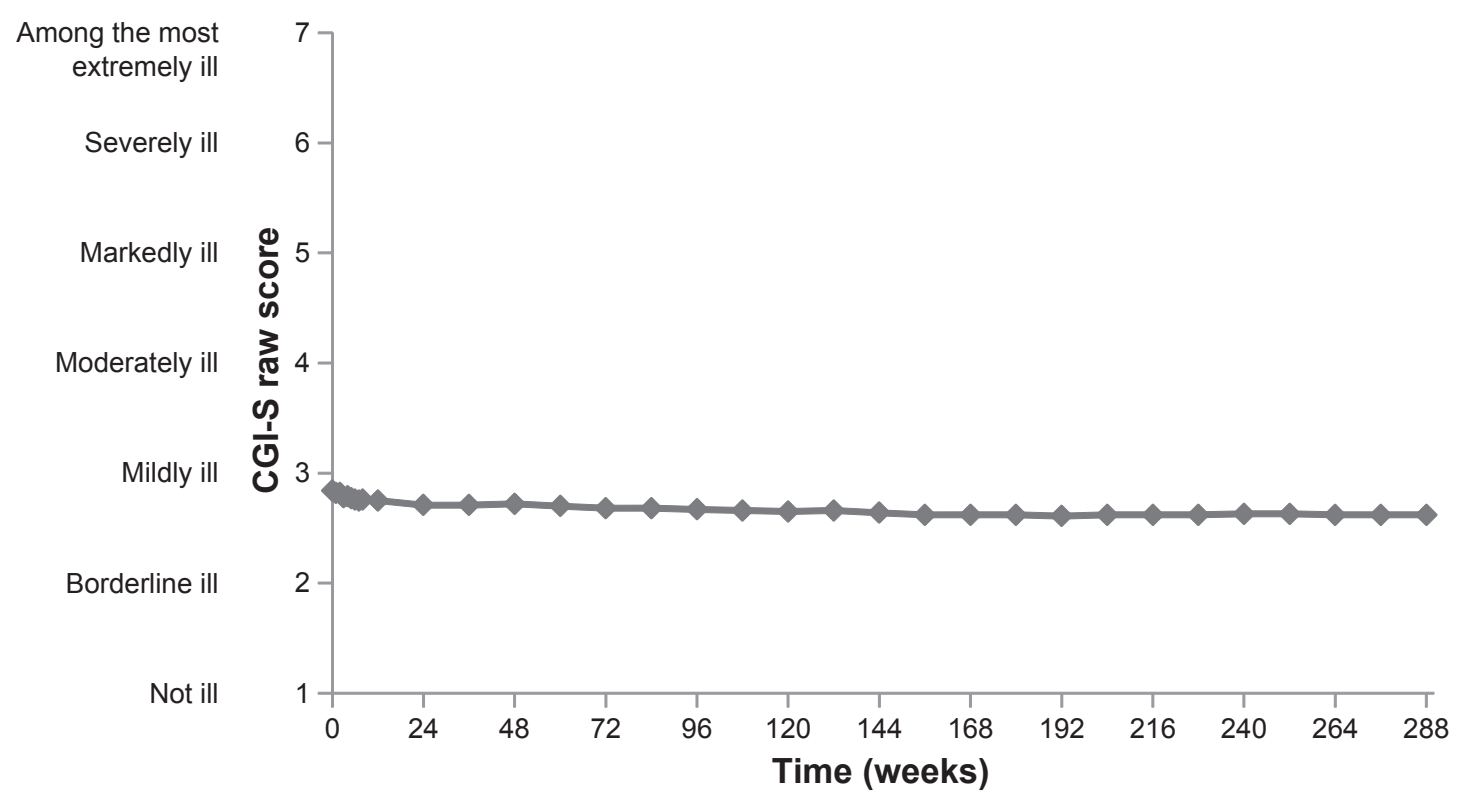

Figure 3 Maintenance of treatment effect. The CGI-S visit-wise mean score for all 669 patients (mean baseline =2.84, mean endpoint $=2.62$ ).

Note: Mean improvements from baseline to endpoint (last observation carried forward) were significant $(P \leq 0.02)$ for all measured time points starting at visit four. Abbreviation: CGI-S, Clinical Global Impression-Severity.

The incidence of injection site-related AEs was 21 (3.1\%). The most frequently reported injection site-related AE was pain, reported in ten patients (1.5\%). All other injection site-related AEs occurred in less than $1 \%$ of patients.

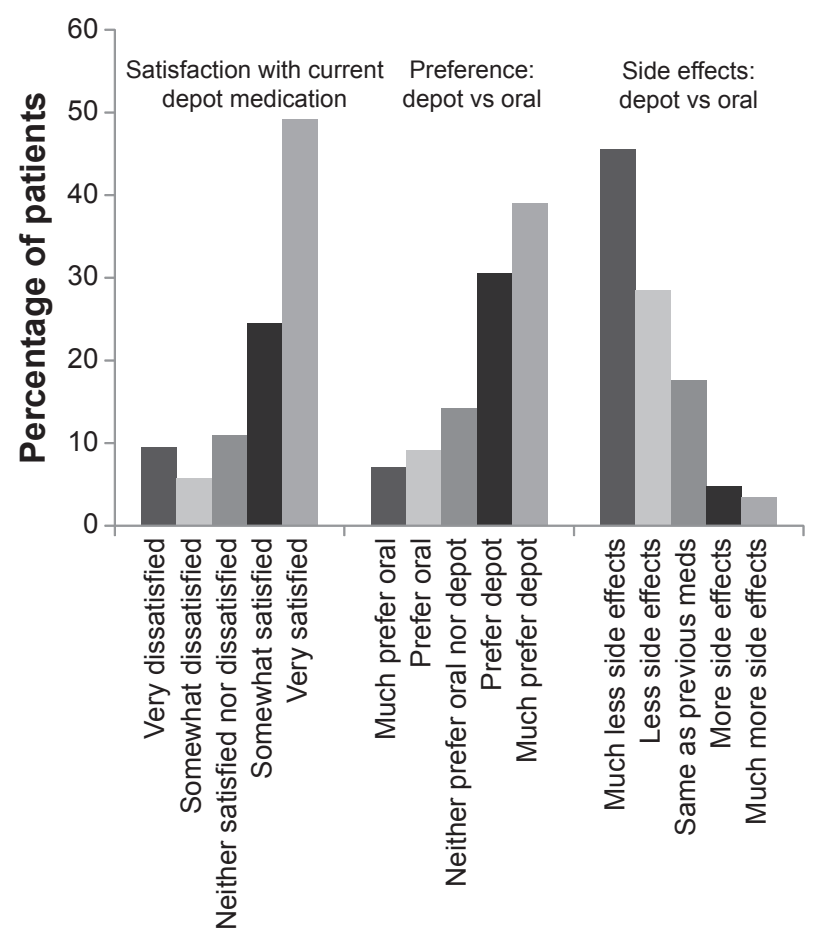

Figure 4 Categorical analysis of the Patient Satisfaction with Medication Questionnaire-Modified at endpoint ( $\mathrm{N}=620)$.

Note: "Depot" refers to olanzapine long-acting injectable.

Abbreviation: N, number of patients.
During the observed period of this post hoc analysis, 24 PDSS events occurred in 23 patients on olanzapine LAI. All patients recovered within 72 hours after receiving the injection, and there were no fatalities. Of the patients who experienced these PDSS events, 19 (82.6\%) continued to receive further injections of olanzapine LAI. Incidence of PDSS per injection was $0.07 \%$.

\section{Laboratory analytes}

Mean changes in metabolic and hepatic laboratory analytes and prolactin are presented in Table 4. A total of $21.1 \%(\mathrm{n}=61)$ of patients developed high fasting prolactin levels $(>1,052.17 \mathrm{pmol} / \mathrm{L}$ for females; $>816.08 \mathrm{pmol} / \mathrm{L}$ for males). Treatment-emergent changes from normal to high ( $\geq 6.99 \mathrm{mmol} / \mathrm{L})$ fasting glucose levels were seen in $2.4 \%$ of patients, changes from normal to impaired

Table 3 Treatment-emergent adverse events in $\geq 5 \%$ of patients

\begin{tabular}{ll}
\hline & $\begin{array}{l}\text { Olanzapine LAI patients } \\
\text { (N=669), } \mathbf{n}(\%)\end{array}$ \\
\hline Patients with $\geq$ I TEAEs & $445(66.5)$ \\
Weight increased & $84(12.6)$ \\
Somnolence & $6 \mathrm{I}(9.1)$ \\
Anxiety & $57(8.5)$ \\
Insomnia & $50(7.5)$ \\
Nasopharyngitis & $43(6.4)$ \\
Headache & $36(5.4)$ \\
\hline
\end{tabular}

Abbreviations: LAl, long-acting injection; $\mathrm{N}$, total number of patients; $\mathrm{n}$, number of affected patients; TEAEs, treatment-emergent adverse events. 
Table 4 Mean changes in laboratory results and weight

\begin{tabular}{|c|c|c|c|c|}
\hline & $\mathbf{N}$ & Baseline mean (SD) & Mean change (SD) & $P$-value \\
\hline Glucose, fasting (mmol/L) & 627 & $5.43(1.35)$ & $0.30(1.59)$ & $<0.001$ \\
\hline Total fasting cholesterol (mmol/L) & 629 & $5.12(1.09)$ & $0.07(0.96)$ & 0.073 \\
\hline HDL fasting cholesterol (mmol/L) & 628 & $\mathrm{I} .14(0.33)$ & $0.04(0.27)$ & $<0.001$ \\
\hline LDL fasting cholesterol (mmol/L) & 623 & $3.12(0.96)$ & $0.03(0.85)$ & 0.427 \\
\hline Triglycerides, fasting (mmol/L) & 629 & $1.94(1.25)$ & $-0.01(1.32)$ & 0.810 \\
\hline Prolactin, fasting (pmol/L) & 410 & $905.21(1,367.82)$ & $14.35(1,163.04)$ & 0.804 \\
\hline Total bilirubin $(\mu \mathrm{mol} / \mathrm{L})$ & 657 & $7.96(4.59)$ & $0.18(3.83)$ & 0.220 \\
\hline Alkaline phosphatase ( $\mu$ kat/L) & 657 & $1.43(0.46)$ & $-0.06(0.34)$ & $<0.001$ \\
\hline AST/SGOT ( $\mu$ kat/L) & 657 & $0.4 I(0.2 I)$ & $-0.01(0.20)$ & 0.293 \\
\hline ALT/SGPT $(\mu k a t / L)$ & 657 & $0.48(0.39)$ & $-0.01(0.35)$ & 0.314 \\
\hline Weight (kg) & 659 & $79.65(17.64)$ & $2.19(8.00)$ & $<0.001$ \\
\hline
\end{tabular}

Abbreviations: ALT/SGPT, alanine transaminase/serum glutamic pyruvic transaminase; AST/SGOT, aspartate aminotransferase/serum glutamic-oxaloacetic transaminase; HDL, high-density lipoprotein; LDL, low-density lipoprotein; N, total number of patients; SD, standard deviation.

$(\geq 5.55 \mathrm{mmol} / \mathrm{L}$ and $<6.99 \mathrm{mmol} / \mathrm{L})$ fasting glucose levels were seen in $20.4 \%$ of patients, and changes from impaired to high fasting glucose levels were seen in $2.9 \%$ of patients. For total cholesterol in fasting state, $3.64 \%$ of patients changed from normal to high $(6.22 \mathrm{mmol} / \mathrm{L})$ and $21.9 \%$ from normal to borderline ( $\geq 5.18 \mathrm{mmol} / \mathrm{L}$ and $<6.22 \mathrm{mmol} / \mathrm{L}$ ). For triglycerides in fasting state, $17.3 \%$ of patients changed from normal to borderline ( $\geq 1.69 \mathrm{mmol} / \mathrm{L}$ and $<2.26 \mathrm{mmol} / \mathrm{L}$ ), $9.3 \%$ changed from normal to high ( $\geq 2.26 \mathrm{mmol} / \mathrm{L}$ ), and $0.6 \%$ changed from normal to very high $(\geq 5.65 \mathrm{mmol} / \mathrm{L})$.

\section{Vital signs, weight, and electrocardiograms}

Statistically significant mean increases were noted for standing (3.69 $\mathrm{mmHg}, \mathrm{SD}=14.18, P<0.001)$ and supine (4.02 mmHg, $\mathrm{SD}=13.37, P<0.001)$ systolic blood pressure, standing $(1.84 \mathrm{mmHg}, \mathrm{SD}=10.09, P<0.001)$ and supine (2.36 mmHg, $\mathrm{SD}=9.68, P<0.001)$ diastolic blood pressure, standing ( 1.35 beats per minute [bpm], $\mathrm{SD}=13.12, P=0.009)$ and supine $(1.83 \mathrm{bpm}, \mathrm{SD}=12.38, P<0.001)$ pulse rate, and temperature $\left(0.08^{\circ} \mathrm{C}, \mathrm{SD}=0.51, P<0.001\right)$, but were not considered to be clinically significant. Baseline-to-endpoint mean change for weight was $2.19 \mathrm{~kg}(\mathrm{SD}=8.00, P<0.001)$. During the observed period of this post hoc analysis, 269 patients (40.8\%) experienced an increase of $\geq 7 \%$ of baseline body weight, whereas 137 patients $(20.8 \%)$ experienced a decrease of $\geq 7 \%$ of baseline body weight.

Mean QT interval, when corrected using the Fridericia method (QTcF), was unchanged (QTcF; $0.67 \mathrm{~ms}, P=0.289$ ), and few patients $(0.3 \%)$ experienced QTcF increases $\geq 60 \mathrm{~ms}$. A decrease in RR interval $(-15.00, P=0.009)$ and an increase in heart rate $(1.38 \mathrm{bpm}, P=0.006)$ were noted. However, there were no clinically significant cardiac events noted with these changes.

\section{EPS}

Rates of EPS were low, with few reports of Parkinsonism (7.1\%), akathisia (3.2\%), or dyskinesia (2.6\%). Mean changes on the individual EPS scales were small (SAS: -0.18 [SD =1.40, $P=0.002]$; BAS: -0.02 [SD $=0.41, P=0.327$ ]; and AIMS: -0.11 [SD =1.45, $P=0.059]$ ). Two patients discontinued treatment after development of tardive dyskinesia. There were no reported cases of neuroleptic malignant syndrome.

\section{Discussion}

The 6-year open-label study on which this post hoc analysis is base ${ }^{10}$ is the longest clinical trial to date designed to evaluate the efficacy and safety of olanzapine LAI, and provides evidence that the efficacy of olanzapine LAI is maintained for the duration of the observed period. For the purposes of the post hoc analysis presented here, 262 patients who participated in the original study ( $\mathrm{N}=931)$ were not included because they were diagnosed with schizoaffective disorder or received supplemental oral olanzapine in amounts greater than that which is consistent with the current recommendations of the European label. ${ }^{11}$ Results of this analysis are generally similar to those seen in the original McDonnell et $\mathrm{a}^{10}$ study as well as with the known efficacy and safety profile of oral olanzapine, ${ }^{2}$ with the exception of injection site-related AEs and PDSS events.

Because the original McDonnell et a ${ }^{10}$ study was uncontrolled, no definitive statements can be made regarding the efficacy of olanzapine LAI per se. However, most of the patients in that study were feeder participants from doubleblind, placebo-controlled trials of olanzapine LAI; ${ }^{5,6}$ hence, most were already being treated for their schizophrenia (though some patients were receiving placebo or subtherapeutic doses of olanzapine LAI). Therefore, the analyses carried out in the original McDonnell et a ${ }^{10}$ study and this 
post hoc analysis were designed to evaluate maintenance of the treatment effect.

PANSS scores (total, positive, negative, and general psychopathology) changed little over the course of the observed period of this post hoc analysis. Similarly, improvements in CGI-S total scores remained stable over the course of the observed period of this post hoc analysis and were not clinically significant. Consistent with this rather stable clinical picture, most patients were satisfied with olanzapine LAI, preferring it to their previous oral medication and experiencing fewer AEs. This is in good agreement with previous research showing that patients taking LAIs favored them over oral medications ${ }^{19}$ and that attitudes about LAI medications were positively correlated with exposure to them. ${ }^{20}$

Because schizophrenia is a chronic mental health condition, the use of long-acting injectable medications has been one strategy applied to help ensure long-term treatment compliance. Despite the length of the study, $44.5 \%$ of patients remained on olanzapine LAI, with a mean treatment duration of approximately 3 years.

With respect to AEs and safety associated with olanzapine LAI, $40.8 \%$ of patients experienced potentially significant weight gain (mean $=2.19 \mathrm{~kg}$ ). Other clinically significant changes were also noted in fasting prolactin, fasting glucose, fasting cholesterol, and fasting triglycerides, which again are generally consistent with the known profile of oral olanzapine. Minor (ie, not clinically significant) changes in blood pressure and heart rate were observed. However, there were no clinically significant cardiac events associated with any of these changes. As previously reported, pharmacokinetic analyses did not reveal any systemic accumulation of olanzapine in this group of patients. ${ }^{10}$ Finally, as expected, rates of EPS were low.

Injection site-related AEs occurred in a somewhat smaller percentage of patients in this post hoc analysis compared to that determined by a pooled analysis of olanzapine LAI clinical trials (3.1\% vs 5.3\%). ${ }^{21}$ It is possible that this lower rate occurred because more patients who experienced injection site-related AEs tended to drop out during the acute trials.

PDSS events (characterized by signs of delirium or excessive sedation, or both) associated with olanzapine LAI in this post hoc analysis occurred at the same rate (events per injection) as that previously reported $(0.07 \%) .{ }^{3}$ All patients recovered from the PDSS events and the majority of patients continued treatment with olanzapine LAI. Because of the risk of PDSS, there are strict regulatory requirements that mandate a number of safety precautions that must be implemented to help mitigate this risk and as fully described in all olanzapine LAI regulatory product-labeling documents. ${ }^{11,22}$

\section{Limitations}

The open-label design and lack of placebo or active comparators in the original McDonnell et al ${ }^{10}$ study, and thus concomitantly in this post hoc analysis, make it difficult to draw detailed conclusions about the relative efficacy or safety of olanzapine LAI from these results. In addition, patients from the three feeder studies had different baseline symptom severities (treatment groups of the feeder studies included placebo and very low doses of olanzapine LAI [45 mg/4 weeks]). Finally, because one purpose of the design of the original McDonnell et $\mathrm{al}^{10}$ study was to mimic real-world conditions, concomitant medications were permitted during that study, thus confounding the evaluation of safety signals.

\section{Conclusion}

The results presented here provide long-term efficacy and safety information for olanzapine LAI when used for the treatment of schizophrenia in a manner that is consistent with the current recommendations of the European label. The reason(s) why these results do not appear to be markedly different from those from the broader patient profile is a potential subject of future research.

With respect to efficacy, patients showed very little change in PANSS total scores and CGI-S scores, suggesting that the long-term maintenance of treatment effect with olanzapine LAI was being clinically successful. Significant decreases in these scores were not expected because patients entered directly from feeder studies in which most were already being treated adequately for their schizophrenia. Patient satisfaction with olanzapine LAI was high.

Safety information observed in this post hoc analysis is generally consistent with the known safety profile of oral olanzapine with the exception of injection site-related and PDSS events. Significant increases in fasting prolactin, fasting glucose, fasting cholesterol, fasting triglycerides, and weight were noted. PDSS events occurred in approximately $0.07 \%$ of injections. Previous analysis of plasma concentrations of olanzapine LAI in these patients revealed no evidence of systemic accumulation.

In conclusion, olanzapine LAI appears to be a safe and effective long-term treatment option for schizophrenia, especially for those patients where adherence and treatment compliance could be a clinical issue. 


\section{Acknowledgments}

This research was funded by Eli Lilly and Company, Indianapolis, Indiana, USA. Assistance with drafting/editing of the manuscript was provided by inVentiv Health Clinical, funded by Eli Lilly and Company. Trial Registration: http:// www.clinicaltrials.gov Identifier: NCT00088465.

\section{Disclosure}

All authors are employees of Eli Lilly and Company. David P McDonnell's current affiliation is Alkermes, Dublin, Ireland. Drs Anand, Tóth, and McDonnell are also shareholders of Eli Lilly and Company. The authors report no other conflicts of interest in this work.

\section{References}

1. Kane JM, Aguglia E, Altamura AC, et al. Guidelines for depot antipsychotic treatment in schizophrenia. European Neuropsychopharmacology Consensus Conference in Siena, Italy. Eur Neuropsychopharmacol. 1998; 8(1):55-66.

2. Hasan A, Falkai P, Wobrock T, et al; WFSBP Task force on Treatment Guidelines for Schizophrenia. World Federation of Societies of Biological Psychiatry (WFSBP) guidelines for biological treatment of schizophrenia, part 2: update 2012 on the long-term treatment of schizophrenia and management of antipsychotic-induced side effects. World J Biol Psychiatry. 2013;14(1):2-44.

3. Detke HC, McDonnell DP, Brunner E, et al. Post-injection delirium/ sedation syndrome in patients with schizophrenia treated with olanzapine long-acting injection, I: analysis of cases. BMC Psychiatry. 2010; $10: 43$.

4. Frampton JE. Olanzapine long-acting injection: a review of its use in the treatment of schizophrenia. Drugs. 2010;70(17):2289-2313.

5. Kane JM, Detke HC, Naber D, et al. Olanzapine long-acting injection: a 24-week, randomized, double-blind trial of maintenance treatment in patients with schizophrenia. Am J Psychiatry. 2010;167(2):181-189.

6. Lauriello J, Lambert T, Andersen S, Lin D, Taylor CC, McDonnell D. An 8-week, double-blind, randomized, placebo-controlled study of olanzapine long-acting injection in acutely ill patients with schizophrenia. $J$ Clin Psychiatry. 2008;69(5):790-799.

7. McDonnell DP, Detke HC, Bergstrom RF, et al. Post-injection delirium/ sedation syndrome in patients with schizophrenia treated with olanzapine long-acting injection, II: investigations of mechanism. BMC Psychiatry. 2010;10:45.
8. McDonnell DP, Kryzhanovskaya LA, Zhao F, Detke HC, Feldman PD Comparison of metabolic changes in patients with schizophrenia during randomized treatment with intramuscular olanzapine longacting injection versus oral olanzapine. Hum Psychopharmacol. 2011; 26(6):422-433.

9. Davis JM. The use of depot medications in the treatment of schizophrenia. Am J Psychiatry. 2010;167(2):125-126.

10. McDonnell DP, Landry J, Detke HC. Long-term safety and efficacy of olanzapine long-acting injection in patients with schizophrenia or schizoaffective disorder: a 6-year, multinational, single-arm, open-label study. Int Clin Psychopharmacol. 2014;29(6):322-331.

11. ZypAdhera. Summary of Product Characteristics; 2013. Accessed from: http://www.ema.europa.eu/docs/en_GB/document_library/ EPAR_-_Product_Information/human/000890/WC500054429.pdf. Accessed March 14, 2015.

12. American Psychiatric Association. Diagnostic and Statistical Manual of Mental Disorders. 4 ed. Washington, DC: American Psychiatric Association; 2000. [text revision]

13. Kay SR, Fiszbein A, Opler LA. The positive and negative syndrome scale (PANSS) for schizophrenia. Schizophr Bull. 1987;13(2):261-276.

14. Guy W. ECDEU Assessment Manual for Psychopharmacology. Rockville, MD: U.S. Department of Health, Education, and Welfare; 1976.

15. Kalali A. Patient satisfaction with, and acceptability of, atypical antipsychotics. Curr Med Res Opin. 1999;15(2):135-137.

16. Simpson GM, Angus JW. A rating scale for extrapyramidal side effects. Acta Psychiatr Scand Suppl. 1970;212:11-19.

17. Barnes TR. A rating scale for drug-induced akathisia. Br J Psychiatry. 1989;154:672-676.

18. Leucht S, Kane JM, Kissling W, Hamann J, Etschel E, Engel R. Clinical implications of brief psychiatric rating scale scores. Br J Psychiatry. 2005;187:366-371.

19. Walburn J, Gray R, Gournay K, Quraishi S, David AS. Systematic review of patient and nurse attitudes to depot antipsychotic medication. Br J Psychiatry. 2001;179:300-307.

20. Waddell L, Taylor M. Attitudes of patients and mental health staff to antipsychotic long-acting injections: systematic review. BrJ Psychiatry Suppl. 2009;52:S43-S50.

21. Atkins S, Detke HC, McDonnell DP, Case MG, Wang S. A pooled analysis of injection site-related adverse events in patients with schizophrenia treated with olanzapine long-acting injection. BMC Psychiatry. $2014 ; 14: 7$

22. Zyprexa Relprevv [US Package Insert]. Indianapolis, IN: Eli Lilly and Company; 2011.
Neuropsychiatric Disease and Treatment

\section{Publish your work in this journal}

Neuropsychiatric Disease and Treatment is an international, peerreviewed journal of clinical therapeutics and pharmacology focusing on concise rapid reporting of clinical or pre-clinical studies on a range of neuropsychiatric and neurological disorders. This journal is indexed on PubMed Central, the 'PsycINFO' database and CAS,

\section{Dovepress}

and is the official journal of The International Neuropsychiatric Association (INA). The manuscript management system is completely online and includes a very quick and fair peer-review system, which is all easy to use. Visit http://www.dovepress.com/testimonials.php to read real quotes from published authors. 